EPJ Web of Conferences 107,10005 (2016)

DOI: $10.1051 /$ epjconf/201610710005

(C) Owned by the authors, published by EDP Sciences, 2016

\title{
Soft photon yield in nuclear interactions
}

\author{
E. Kokoulina ${ }^{1,2, a}$, on behalf of the SVD-2 Collaboration \\ ${ }^{1}$ JINR, Dubna, Russia \\ ${ }^{2}$ GSTU, Gomel, Belarus
}

\begin{abstract}
First results of the study of a soft photon yield in nucleus-nucleus collisions at $3.5 \mathrm{GeV}$ per nucleon at Nuclotron (LHEP, JINR) are presented. These photons are registered by an BGO electromagnetic calorimeter built by the SVD-2 Collaboration. The obtained spectra confirm the excessive yield in the energy region less than $50 \mathrm{MeV}$ in comparison with theoretical estimations and agree with previous experiments at high-energy interactions.
\end{abstract}

\section{Introduction}

Physicists consider that at interactions of nuclei with proton and deuteron beams are formed cold nuclear matter [1]. These researches permit to compare properties of hot quark-gluon medium formed in collisions of relativistic heavy ions and cold nuclear matter producing in $p p$ or $p(d) \mathrm{A}$ interactions. The SVD-2 Collaboration carries out studies of $p p, p \mathrm{~A}$ and AA interactions. These experiments are fulfilled at U-70 in IHEP, Protvino with $50 \mathrm{GeV}$-proton beams [2] and at Nuclotron (JINR) with $3.5 \mathrm{GeV} /$ nucleon nucleus beams.

The SVD-2 Collaboration investigates the unique region of high multiplicity in which a number of collective phenomena of secondary particles is predicted. The following results have been received[2, 3]:

* the topological cross sections in the region up to charged multiplicity $N_{c h}=24$ that had permitted to advance in them down on three orders of magnitude;

* the average multiplicity and variance of number of charged particles;

* distributions on the number of neutral pions at fixed total pion multiplicity, $N_{t o t}=N_{c h}+N_{0}\left(N_{0}-\right.$ number of $\pi^{0}$-mesons);

* the rapid growth of the scaled variance, $\omega=D / \bar{N}_{0}$, with the increasing of total pion multiplicity $(D-$ a variance of the neutral meson number at the fixed total multiplicity, $\bar{N}_{0}$ - their mean multiplicity).

The growth of the experimental value $\omega$ gets 7 standard deviations in respect of Monte Carlo predictions. This result is one of the evidences of the Bose-Einstein condensate (BEC) formation [4].

The theoretical description of this collective phenomenon has been developed by Begun and Gorenstein [4] at the specific conditions of the SVD-2 experiment at $\mathrm{U}-70$ in $p p$ interactions. They have estimated the total pion multiplicity at which BEC can start to form. The

\footnotetext{
a e-mail: kokoulin@sunse.jinr.ru
}

German physicist S. Barshay predicts [5] that the pion condensation may be accompanied by an increased yield of soft photons (SP) with energy smaller than $50 \mathrm{MeV}$. The anomalous SP have being studied experimentally during more than 30 years [6-9]. There are some theoretical models worked out to explain the SP yield [10-12]. Unfortunately, incompleteness of data does not permit disclosing of the physical essence of this phenomenon.

To understand nature of the SP formation comprehensively and, in particular, to test relation between the SP excess yield and the BEC formation, the SP electromagnetic calorimeter (SPEC) has been manufactured and tested by the SVD Collaboration at U-70 accelerator [13]. This calorimeter is a stand-alone device and it differs from many similar ones by its extremely low energy threshold of gamma-quantum registration - of order of $2 \mathrm{MeV}$. The SPEC technique permits to execute the unique research program of $p p, p \mathrm{~A}$ and $\mathrm{AA}$ interactions with registration of SP.

The report is organized in the following way. The few previous SP experiments are reviewed in section 2. In section 3 the description and technical characteristics of the electromagnetic calorimeter manufactured by the SVD Collaboration are given. The first preliminary spectra of SP obtained with the deuterium and lithium beams on a carbon target at Nuclotron are also presented in this section.

\section{Review of some experiments recording soft photons}

Experimental and theoretical studies of direct photon production in hadron and nuclear collisions essentially expand our knowledge about multi-particle production mechanisms. These photons are useful probes for investigation of nuclear matter at all stages of the interaction. The SP play a particular role in these studies. Until now we 
have no complete explanation of the experimentally observed excess of the SP yield. These soft photons have low transverse momenta $p_{T}<0.1 \mathrm{GeV} / \mathrm{c}$ and Feynman variable $|\mathrm{x}|<0.01$. In this domain their yield exceeds the theoretical estimates by $3 \div 8$ times [6-9].

This anomalous phenomenon has been discovered at the end of 1970s with the Big Europe Bubble Chamber at the SPS accelerator, in CERN, in the experiment with $70 \mathrm{GeV} / \mathrm{c} \mathrm{K}^{+}$-meson and antiproton beams [6]. The SP yield has exceeded the theoretical predictions by $4.5 \pm 0.9$ times. The following electronic experiments [7-9] have confirmed an anomalous behaviour of the SP.

The WA83 Collaboration studied the direct SP yield at OMEGA spectrometer in $\pi^{-}+p$ interactions at hydrogen target with $280 \mathrm{GeV} / c \pi^{-}$-mesons. Excess yield of the SP turned out to be equal to $7.9 \pm 1.4$ [7]. The last experimental study of SP has been carried out at the LEP accelerator with DELPHI setup in CERN [9].

Two kinds of processes were investigated in this experiment: $e^{+}+e^{-} \rightarrow Z^{0} \rightarrow$ jet $+\gamma$ and $e^{+}+e^{-} \rightarrow \mu^{+}+\mu^{-}$. In processes with formation of hadron jets the DELPHI Collaboration has revealed surplus of the SP yield over Monte Carlo estimations at the level $4.0 \pm 0.3 \pm 1.0$ times. For the first time the SP yield at maximum number of neutral pions 7-8 has amounted to about 17-fold excess [9] in comparison with bremsstrahlung of charged particles. On the contrary, in the lepton process without formation of hadron jets the yield of SP turned out to agree well with theoretical predictions.

The theoretical models try to explain the anomalous SP yield. The SVD-2 Collaboration developed a gluon dominance model [12] explained the excessive SP yield by the production of soft gluons in quark-gluon system. These gluons have no enough energy to fragment into hadrons, so they are scattered on the valency quarks of secondary particles and form the SP [12]. This model gives twothree-fold exceed over common accepted area of strong interactions in accordance with estimations of the region of SP emission [12].

\section{Technical characteristics of SPEC. Experimental spectra of soft photons}

SPEC has been manufactured on the base of the BGO scintillators (bismuth ortogermanate) [13]. The BGO crystals have a small radiation length $\mathrm{X}_{0}=1.12 \mathrm{~cm}$ (for comparison, crystals $\mathrm{NaI}(\mathrm{Tl})$ have $\left.\mathrm{X}_{0}=2.59 \mathrm{~cm}\right)$, that permits to reduce considerably the volume of the device. Moreover, this scintillator has small sensibility to neutrons which is important at the measurement of a gamma-radiation. At manufacturing of such calorimeter the problems of uniform distributions of activator in the crystal volume do not appear. Whereas many of inorganic scintillators have the long-term of emission, BGO crystals show relatively small afterglow.

The scheme of SPEC is shown in Fig. 1 (at the top). It is a square matrix composed of $49(7 \times 7)$ counters. The counter with a demultiplier and a preliminary amplifier are placed directly on the panel PMT as shown in Fig. 1

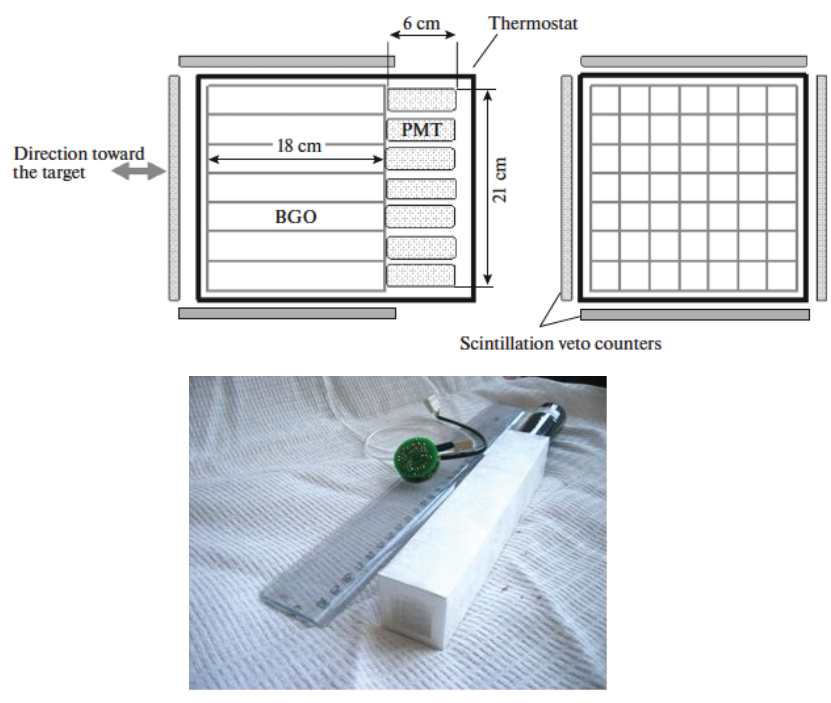

Figure 1. (Color online) Top panel: scheme of SPEC. Bottom panel: BGO crystal with PMT.

(below) [13]. Every crystal has the parallelepiped form, $30 \times 30 \times 180 \mathrm{~mm}^{3}$. $180 \mathrm{~mm}$ correspond to 16 radiation lengths. Its front side is covered by the high-reflective film VM2000. The lateral facets of crystals are wrapping up of Tyvic for increasing of light gathering (the thickness $120 \mu \mathrm{m}$ ). The PMT 9106SB are used (ET Enterprises). They have 8 dynodes and high quantum efficiency in the green part of spectrum. The photocathode diameter is equal to $25 \mathrm{~mm}$. The tube has the permalloy magnetic protection. PMT is glued to the crystal by the optic EPOTEK 301 glue.

The box with counters is surrounded by the scintillator detectors of a guard veto-system. SPEC is placed inside the thermostat (the top panel of Fig. 1). The thermo stabilization is realized by the cooling system Huber 006B. The temperature of liquid in the thermostat can change from $-20^{\circ}$ up to $+40^{\circ} \mathrm{C}$. After few trials the temperature was chosen to be $+18^{\circ} \mathrm{C}$.

The plastic veto-detector of charged particles $\left(23 \times 23 \times 1 \mathrm{~cm}^{3}\right)$ is placed before the crystals. Behind it an assembly of 4 plastics of a pre-shower $\left(18 \times 4.5 \times 1 \mathrm{~cm}^{3}\right)$ is shown in Fig. 2 (at the top). A lead $2 \mathrm{~mm}$-convertor is put between the front-veto and plastics. In this figure, the target and counters of a trigger system are shown also. The trigger is produced at the signal from any 2 of 4 pre-shower counters. There are two large veto-counters in front of the target. They are necessary to forbid a response from the beam halo. SPEC with veto-detectors was laid at Nuclotron hall near the NIS-GIBS setup (Fig. 2, bottom panel).

The maximum of the signal-noise ratio is provided by input capacitance minimization. It is determined by the dynode-anode gap and capacitance of assembly that amounts to $\sim 6 \mathrm{pF}$. The noise in the spectrometric channels does not exceed $100 \mathrm{keV}$ that permits for the first time measuring of the SP spectrum in the range $0.6 \div 600 \mathrm{MeV}$. The dynamic diapason of signals amounts to more than 

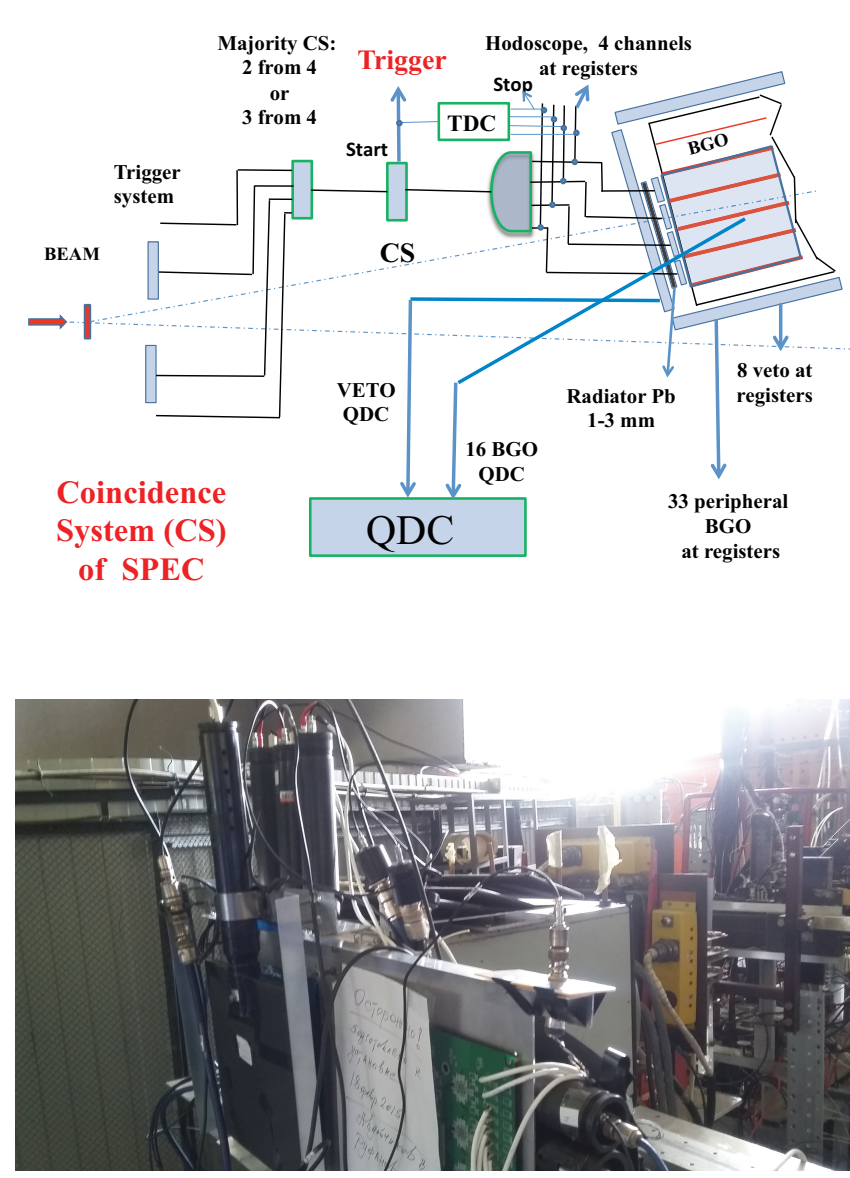

Figure 2. (Color online) Top panel: the reductive working scheme of SPEC at the Nuclotron. Bottom panel: view of SPEC with veto-detectors at NIS-GIBS setup.

$66 \mathrm{~dB}$. Time-stamp is given by the $4.5 \times 4.5 \times 0.1 \mathrm{~cm}^{3}$ beam counter (not shown). It is also placed in front of the target.

During the Nuclotron run, SPEC is set at an angle of $16^{\circ}$ relative to the beam direction. The front plane of crystals is away from the target at the distance $203 \mathrm{~cm}$. The digitization of plastic scintillators is realized with CAMAC ADCs (Lecroy 2249A) and TDCs (LeCroy 2228A), the digitization of analog signals of calorimeter - by ADC CC-008.

We used CAMAC and a LE-88K crate-controller with input for a trigger signal. The crate-controller was connected to PC with PCI-QBUS interface. Data acquisition software has been developed in MIDAS framework (http://midas.psi.ch). Time of flight between the beam counter and the pre-shower for neutral particles (no signal in the front-veto) gave time resolutions $632 \mathrm{ps}$ for $\mathrm{d}+\mathrm{C}$ and 532 ps for $\mathrm{Li}+\mathrm{C}$ interactions (Fig. 3).

In 2014 and 2015 two experimental runs (50th and 51 st, respectively) were carried out at Nuclotron in LHEP JINR with $3.5 \cdot \mathrm{A} \mathrm{GeV}$ deuterium (18 hours) and lithium (14 hours) beams. SPEC has been installed at the location of NIS-GIBS setup.
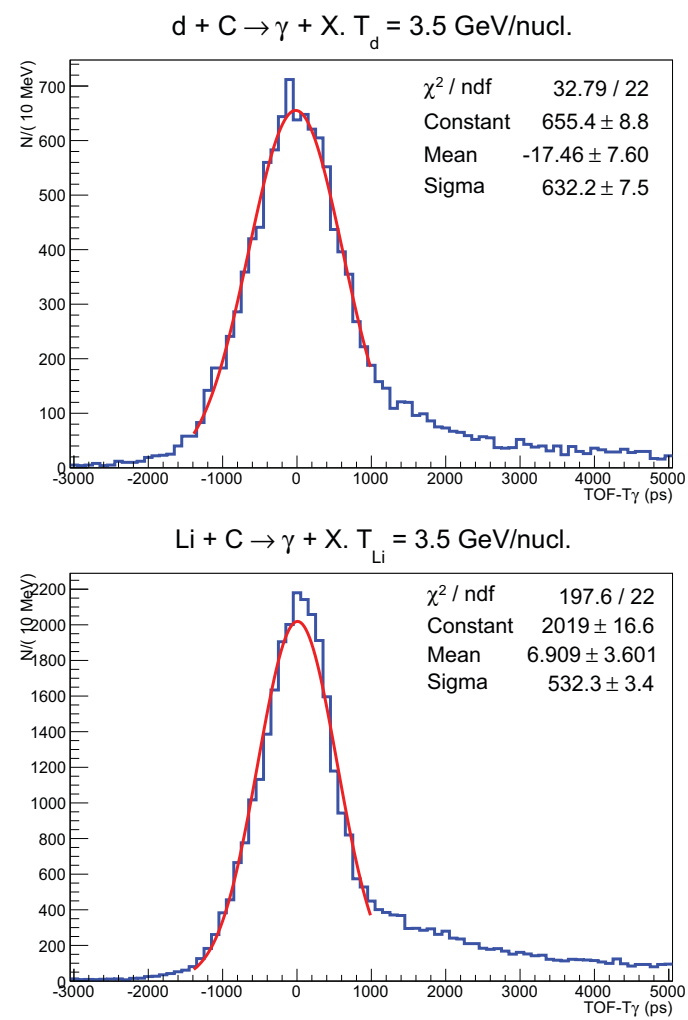

Figure 3. (Color online) Top panel: time resolution in preshower for $\mathrm{d}+\mathrm{C}$ interactions. Bottom panel: the same as above for $\mathrm{Li}+\mathrm{C}$ interactions.
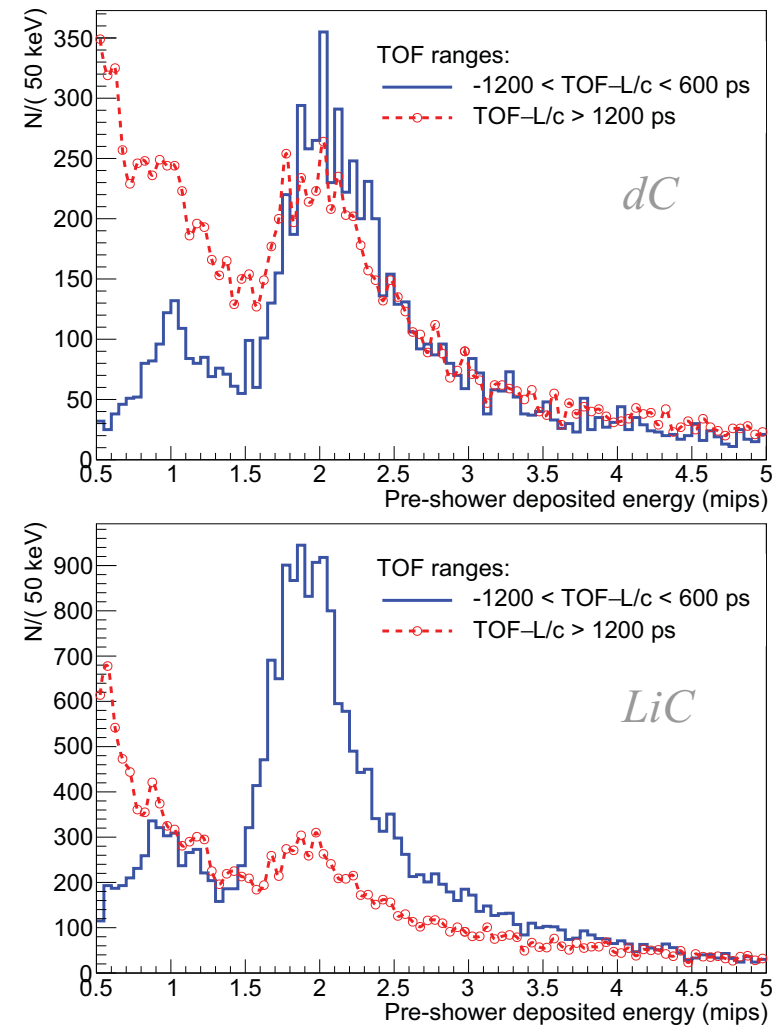

Figure 4. (Color online) Top panel: time of flight of neutral particles between the beam counter and the pre-shower for $\mathrm{d}+\mathrm{C}$ interactions. Bottom panel: the same as above for $\mathrm{Li}+\mathrm{C}$ interactions. 

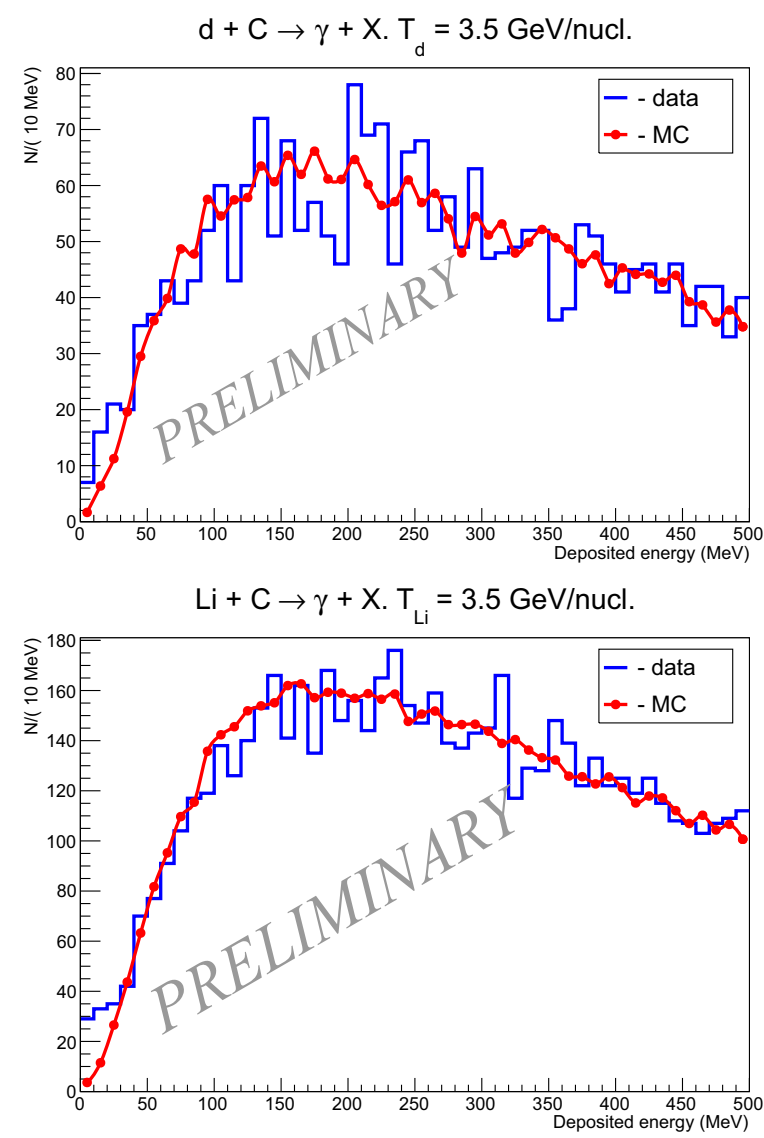

Figure 5. (Color online) Entire energy spectra in SPEC with preshower and simulation in $\mathrm{d}+\mathrm{C}$ (top panel) and in $\mathrm{Li}+\mathrm{C}$ (bottom panel) interactions at Nuclotron.

Criterions of event selection were the following:

1) energy in the front veto-counter smaller than 0.3 MIPs

2) energy in pre-shower $0.5<E<4$ MIPs;

3) time of flight $-1200<\mathrm{t}-\mathrm{t}_{\gamma}<600 \mathrm{ps}$;

4) more than $2 \mathrm{MeV}$ is registered in one of BGO crystals;

5) location of shower in BGO crystal must overlay throughout vertical with the triggered pre-shower counter;

6) energy deposition in the outer BGO layer should be no more than $1 / 3$ of a total to prevent significant leakages.

In Fig. 4, spectra of $\gamma$ quanta deposited in pre-shower plastic with time selection for neutral particles is presented for $\mathrm{d}+\mathrm{C}$ (on the top) and $\mathrm{Li}+\mathrm{C}$ (below) interactions. A solid line shows the Compton peak at 1 MIP energy and more intensive peak of gamma quanta conversion at 2 MIP. In this figure with dotted line the structure for $t-t_{\gamma}>1200$ ps is almost unnoticeable.

The Monte Carlo simulation of the SPEC setup was carried out at the conditions of the last assembly and the beam energy $-3.5 \cdot \mathrm{A} \mathrm{GeV}$. The Monte-Carlo simulation (uRQMD+Geant-3.21) was used. Geant-4 shown the same result.

After data processing we obtained the SP spectra of energy release in deuterium-carbon (Fig. 5, top panel) and lithium-carbon (Fig. 5, bottom panel) interactions. In the region of energy below than $50 \mathrm{MeV}$, a noticeable excess over the Monte-Carlo simulation results is observed. It agrees well to other SP experiments [7].

\section{References}

[1] A. Andronic et al., arXiv:1506.03981 [nucl-ex]

[2] V.V. Avdeichikov et al., Proposal "Termalization" (in Russian), Preprint JINR-P1-2004-190, JINR, Dubna (2005)

[3] E. S. Kokoulina, on behalf of the SVD-2 Collaboration, Progr. Theor. Phys., 193, 306 (2012); V. N. Ryadovikov, on behalf of the SVD-2 Collaboration, Phys. At. Nucl.75, 989 (2012)

[4] V.V. Begun and M.I. Gorenstein, Phys. Lett. B 653, 190 (2007); V.V. Begun and M.I. Gorenstein, Phys. Rev. C 77, 064903 (2008)

[5] S. Barshay, Phys. Lett. B 227, 279 (1989)

[6] P.V. Chliapnikov et al., Phys. Lett. B 141, 276 (1984)

[7] S. Banerjee, et al., SOPHIE/WA83. B305, 182 (1993)

[8] J. Schukraft, HELIOS Collaboration, Nucl. Phys. A 498, 79 (1989)

[9] J. Abdallah et al., DELPHI Collaboration, Eur. Phys. J. C 47, 273 (2006)

[10] L. Van Hove, Ann. Phys. (NY), 192, 66 (1989); P. Lichard and L. Van Hove, Phys. Lett. B 245, 605 (1990)

[11] Wong Cheuk-Yin, Phys. Rev. Lett. 81, 064903 (2010)

[12] E. Kokoulina, Acta Phys. Polonica B 35, 295 (2004); M.K. Volkov, E. Kokoulina, and E.A. Kuraev, PEPAN Lett., 5, 16 (2004)

[13] E.N. Ardashev et al., Instr. Exp. Tech. 58, 18 (2015) 